\title{
Neural Mechanisms Underlying Human Auditory Evoked Responses Revealed By Human Neocortical Neurosolver
}

\author{
Carmen Kohl $^{1}$ (D) $\cdot$ Tiina Parviainen $^{2,3}$ (D) . Stephanie R. Jones ${ }^{1,4}$ (D)
}

Received: 29 December 2020 / Accepted: 30 March 2021 / Published online: 19 April 2021

(c) The Author(s) 2021

\begin{abstract}
Auditory evoked fields (AEFs) are commonly studied, yet their underlying neural mechanisms remain poorly understood. Here, we used the biophysical modelling software Human Neocortical Neurosolver (HNN) whose foundation is a canonical neocortical circuit model to interpret the cell and network mechanisms contributing to macroscale AEFs elicited by a simple tone, measured with magnetoencephalography. We found that AEFs can be reproduced by activating the neocortical circuit through a layer specific sequence of feedforward and feedback excitatory synaptic drives, similar to prior simulation of somatosensory evoked responses, supporting the notion that basic structures and activation patterns are preserved across sensory regions. We also applied the modeling framework to develop and test predictions on neural mechanisms underlying AEF differences in the left and right hemispheres, as well as in hemispheres contralateral and ipsilateral to the presentation of the auditory stimulus. We found that increasing the strength of the excitatory synaptic cortical feedback inputs to supragranular layers simulates the commonly observed right hemisphere dominance, while decreasing the input latencies and simultaneously increasing the number of cells contributing to the signal accounted for the contralateral dominance. These results provide a direct link between human data and prior animal studies and lay the foundation for future translational research examining the mechanisms underlying alteration in this fundamental biomarker of auditory processing in healthy cognition and neuropathology.
\end{abstract}

Keywords Auditory processing $\cdot$ AEF $\cdot$ MEG $\cdot$ Biophysical model $\cdot$ HNN

Handling Editor: Jeremie Lefebvre.

This is one of several papers published together in Brain Topography on the "Special Issue: Computational Modeling and M/EEG”.

Carmen Kohl

carmen_kohl@brown.edu

1 Department of Neuroscience, Carney Institute for Brain Sciences, Brown University, Providence, USA

2 Centre for Interdisciplinary Brain Research, Department of Psychology, University of Jyväskylä, P.O. Box 35, 40014 Jyväskylä, Finland

3 Meg Core Aalto Neuroimaging, Aalto University, AALTO, P.O. Box 15100, 00076 Espoo, Finland

4 Center for Neurorestoration and Neurotechnology, Providence VAMC, Providence, USA

\section{Introduction}

Brain activity evoked by auditory stimulation has been studied for many decades and remains not only commonly used in cognitive neuroscience (Wagner et al. 2017; Parviainen et al. 2019) but also clinically relevant (Paulraj et al. 2015; Samatra et al. 2020). In humans, the sequence of neural activation evoked by auditory stimulation can be measured using electroencephalography (EEG) or magnetoencephalography (MEG), and the surface-recorded responses are typically divided into three categories based on their latency. Broadly speaking, early responses (within $\sim 10 \mathrm{~ms}$ ) primarily reflect brain stem activity, middle-latency auditory responses (10-50 ms) are thought to reflect processing in thalamocortical structures, and late-latency responses (50-250 ms) are associated with cortical activity (Picton et al. 1974). Late latency responses are elicited in the primary auditory cortex and surrounding areas and typically consist of components labelled P50m-N100m-P200m (or P1-N1-P2), which peak at around 50, 100 and $180 \mathrm{~ms}$ respectively (Eggermont and 
Ponton 2002). Here, we refer to this cortical P50m-N100mP200m sequence as auditory evoked fields (AEF). Although the P50m-N100m-P200m waveform is commonly found in response to any auditory event, the shape and amplitude of its components depends on a number of characteristics of the evoking stimuli, such as their acoustic complexity, intensity, duration, and frequency (Picton 2011). Here, we use simple pure tones at $1 \mathrm{kHz}$, which have been shown to produce robust N1 responses and are neutral in terms of linguistic associations, and adopted a paradigm that has been successfully used to evidence the fundamental response properties of the human auditory processing pathway (Mäkelä et al. 1994; Salmelin et al. 1999).

While AEFs are commonly used as correlates for a number of cognitive processes (Shahin et al. 2003; Fan et al. 2017), developmental stages (Parviainen et al. 2019; van Bijnen et al. 2019), and clinical observations (Goodin et al. 1978; Stephen et al. 2017), several fundamental questions about their neural origin remain unanswered. A deeper understanding of the cellular and circuit mechanisms generating AEFs is essential to understanding the role of $\mathrm{AEF}$ components in cognition and to developing treatments based on regularizing neuropathological AEF features.

Insights into the biophysical mechanisms underlying auditory processing have been gained using invasive recordings in animal models. An early description of a fundamental anatomical circuit of sensory processing was based on cat visual cortex (Gilbert 1983; Gilbert and Wiesel 1989) and states that excitatory cells in layer IV of the neocortex receive thalamic inputs and project to superficial layers, which in turn project to deeper layers. This fundamental layer specific relay of sensory information has since been confirmed in other sensory systems, including somatosensory (Di et al. 1990; Schroeder et al. 1995) and auditory cortex (Ojima et al. 1991, 1992), with confirmation from a number of studies and species (e.g. Mitani and Shimokouchi 1985; Mitani et al. 1985; Pandya and Rosene 1993; Huang and Winer 2000; Sakata and Harris 2009; Atencio and Schreiner 2010; Ji et al. 2016). Importantly, the commonality of this laminar sequence of sensory information flow in different sensory areas suggests that the basic laminar structure and organization is similar across sensory regions and deterministic of the early sensory response, particularly in primary sensory cortices (Douglas and Martin 2004; Barbour and Callaway 2008; Atencio and Schreiner 2010).

Although animal models provide insight into cell and circuit-level dynamics underlying sensory evoked responses, determining if and how this information translates to macroscale human signals, as measured with EEG and MEG, is a non-trivial problem. In recent years, computational neuroscience has begun to bridge the gap between macroscale human brain signals and network-dynamics (Kiebel et al. 2009; Sanz Leon et al. 2013; Hagen et al. 2018). One approach to achieve this is proposed by the modeling software Human Neocortical Neurosolver (HNN; Neymotin et al. 2020), namely to create a model of neuronal circuitry informed by invasive animal recordings and use this model to simulate human macroscale brain signals based on their biophysical origin. The model underlying HNN consists of a canonical neocortical circuit, with individual excitatory and inhibitory neurons across the cortical layers, and layer specific thalamocortical and cortico-cortical input pathways. The primary electrical currents generating EEG and MEG signals are simulated from the intracellular current flow in the long and spatially aligned cortical pyramidal neuron apical dendrites (for a detailed description, see Methods, supplement S1, or visit hnn.brown.edu). HNN has been applied to study the cellular and circuit level neural mechanism underlying a number of commonly measured EEG and MEG signals, such as alpha and beta frequency oscillations (Jones et al. 2009; Ziegler et al. 2010; Sherman et al. 2016), gamma oscillations (Lee and Jones 2013), and somatosensory evoked fields (SEF; Jones et al. 2007, 2009; Ziegler et al. 2010; Sliva et al. 2018). Here, we build from the prior literature and known commonalities in the cortical structure and information relay in sensory areas to apply HNN to study the circuit mechanisms underlying MEG measured AEFs. We focus on interpreting the neural mechanisms generating (1) the AEF waveform in response to a simple auditory tone (Parviainen et al. 2019), including the P50m-N100m-P200m sequence, (2) observed differences in AEFs in the right and left hemisphere, and (3) between contralateral and ipsilateral tone presentations. We hypothesized that the laminar organization and relay of sensory information is preserved across sensory areas, and that, as a result, AEFs could be simulated using similar cortical input sequences as those shown to reproduce SEFs in our prior studies. Note that, while the laminar organization and exogenous drives simulated in HNN are based on animal work (see Discussion), previous work has shown that the resulting simulations can be applied to human data and support canonical input sequences (Jones et al. 2007, 2009; Ziegler et al. 2010; Sliva et al. 2018; Neymotin et al. 2020). Building from this hypothesis, we then investigated the observed phenomenon that the AEF recorded over the right hemisphere, and specifically the $\mathrm{N} 100 \mathrm{~m}$ component, is often larger in amplitude compared to the left AEF (Peronnet et al. 1974; Mononen and Seitz 1977; Wolpaw and Penry 1977; Hine and Debener 2007; Howard and Poeppel 2009; Kimura 2011; Shaw et al. 2013). The biophysical origin of this difference is unknown, and we apply HNN to develop targeted predictions on neural mechanisms that may generate these differences.

Lastly, we examine the mechanisms underlying AEF differences that depend on the site of auditory stimulation, i.e. between contralateral and ipsilateral responses. In early AEF research, it was found that when simple sounds were 
presented monaurally, responses in the hemisphere contralateral to the sound presentation were more pronounced than responses in the ipsilateral hemisphere (Tunturi 1946; Rosenzweig 1951). This increased magnitude in contralateral auditory responses has since been confirmed in a number of experimental paradigms and recording modalities (Peronnet et al. 1974; Andreassi et al. 1975; Taub et al. 1976; Wolpaw and Penry 1977; Reite et al. 1981; Pantev et al. 1986, 1998; Yoshiura et al. 1994; Loveless et al. 1994; Mäkelä et al. 1994; Jäncke et al. 2002; Devlin et al. 2003; Petkov et al. 2004; Parviainen et al. 2019). In addition, the contralateral response has been described as not only larger in magnitude, but also as faster than its ipsilateral equivalent (Mononen and Seitz 1977; Wolpaw and Penry 1977; Mäkelä et al. 1994; Pantev et al. 1998). However, this temporal effect appears to be less clear than the effect on magnitude, as several studies failed to confirm any temporal differences (Tunturi 1946; Andreassi et al. 1975; Yoshiura et al. 1994).

While there is strong evidence suggesting that the contralateral dominance effect is robust (although there is some debate, e.g. Hine and Debener 2007), few studies have focused on the biophysical mechanisms underlying this phenomenon. These studies guide our neural model investigation of observed contralateral AEF dominance in our data. One explanation for contralateral dominance is that, while auditory processing is not as lateralized as, for example, somatosensory processing, those pathways between ear and cortex which cross over nevertheless appear dominant, with greater numbers of fibers and faster transmission speeds (Rosenzweig 1951; Kimura 1961). This feature is most likely to account for temporal differences, as stronger pathways may lead to faster transmission of information, resulting in earlier contralateral AEF peaks. However, it is not immediately apparent that the integrity of fiber tracts alone necessarily leads to responses of greater magnitude. A second explanation states that larger areas of cortex are activated by contralateral stimulation (Rosenzweig 1951; Gross et al. 1967; Pantev et al. 1986), which would lead to greater amplitude responses. Gross et al. (1967) found that the cortical area in which responses could be evoked was larger when tones were presented contralaterally as opposed to ipsilaterally. Importantly, these explanations are based on evidence almost exclusively from animal models, and it is unknown if they can account for the contralateral dominance observed in human macroscale AEFs. We therefore applied our HNN modeling framework to test the hypothesis that changes in model parameters that represent the speed of activation of the cortex, and number of cells contributing to the signal, could account for the observed differences in contralateral vs. ipsilateral MEG measured AEFs.

In summary, the goals of the current study were threefold. (1) We aimed to simulate an AEF waveform in HNN and hypothesized that the cortical inputs underlying the waveform share important features with those previously shown to be involved in other sensory evoked responses, due to the canonical structure of sensory neocortex. (2) We used HNN to explore which biophysical mechanisms underlie the commonly observed difference between right hemisphere and left hemisphere AEFs. (3) We tested the hypothesis that differences in the scale of the underlying network as well as the latencies of the inputs into the network could account for the previously reported dominance of contralateral AEFs.

\section{Methods}

\section{Participants}

Participant recruitment, MEG recording and source-localization were performed for a previous study. For a more detailed description, see Parviainen et al. $(2005,2019)$.

Ten neurotypical participants (age 23-39; five females) were recruited for MEG recording.

\section{Auditory Stimulation}

Participants were presented with simple $1 \mathrm{kHz}$ sine wave tones, $50 \mathrm{~ms}$ in duration with $10 \mathrm{~ms}$ fade-in and fade-out time, created in Sound Edit (MacroMedia, San Francisco, CA, USA). The tones were presented alternatingly to the left and right ear (Parviainen et al. 2019). Tones were separated by inter-stimulus-intervals varying between 0.8 and $1.2 \mathrm{~s}$ and were presented at $60 \mathrm{~dB}$ above the subjective hearing level.

\section{MEG Acquisition and Analysis}

MEG was recorded using a helmet-shaped 306-channel whole-head system (Vectorview, Neuromag Ltd, Helsinki, Finland), band-pass filtered at $0.03-200 \mathrm{~Hz}$ and sampled at $600 \mathrm{~Hz}$. Segments of $-200 \mathrm{~ms}$ to $800 \mathrm{~ms}$ relative to auditory stimulus onset were averaged off-line for each participant, separately for left and right ear sounds. Electro-oculogram (EOG) was recorded and epochs in which the EOG channel exceeded a threshold of $150 \mu \mathrm{V}$ were considered contaminated by blinks or saccades, and were excluded from the averages. After artifact rejection, an average of 102 $( \pm 4) / 105( \pm 4)($ mean $\pm S D)$ epochs remained per subject for left/right ear sounds (see Parviainen et al. 2005, 2019).

The resulting averages were source-localized using equivalent current dipole (ECD) modeling, where ECDs represent the average distribution of electric current within a cortical patch, giving an estimate of the location, strength, and direction of local current flow (Hämäläinen et al. 1993). For determining the ECD, the temporally varying field pattern was visually inspected between 80 and $120 \mathrm{~ms}$ relative to the auditory stimulus to identify symmetric dipolar fields, 
indicative of separable, active neuron population. The $\mathrm{N} 100 \mathrm{~m}$ peak visible at the sensor-level was always associated with salient dipolar field pattern (cf. Fig. 2d, e). ECDs were determined for each participant (one dipole per hemisphere) from a standard subset of 46 planar gradiometers that covered the $100 \mathrm{~ms}$ auditory field pattern (Fig. 2). The determined ECDs in each hemisphere were then used to account for the MEG signals by keeping ECD location and orientations fixed and varying only their amplitude. For further analysis in this study, source-localized AEFs were segmented into $0 \mathrm{~ms}$ to $250 \mathrm{~ms}$ epochs, relative to the auditory stimulus, and averaged across participants. The same pair of ECDs, at an individual level, accounted for the activation patterns evoked by both ipsilateral and contralateral stimulation. Moreover, the ECD that was fitted to the $100 \mathrm{~ms}$ response (N100m) was also able to account for the preceding response at $50 \mathrm{~ms}$ (P50m). The current direction of the $\mathrm{N} 100 \mathrm{~m}$ peak was into the cortex, and the P50m and P200m have amplitudes in the opposite direction representing current out of the cortex.

Source localization is an important step since we intended to model the AEF waveforms using the Human Neocortical Neurosolver software (HNN, see below), which is designed to study the origin of source localized signals. Inverse solution methods applied to sensor-level MEG (or EEG) estimate the primary current generator of the sensor data. These primary currents are estimated as current dipoles in units of current $x$ distance (Ampere $\times$ meters) and known to be generated by intracellular post-synaptic current flow in the long and spatially aligned cortical pyramidal neuron dendrites (Ikeda and Shibasaki 1992; Hämäläinen et al. 1993; Okada et al. 1997; Murakami et al. 2003; Murakami and Okada 2006; Sacchet et al. 2015; Neymotin et al. 2020). As detailed below, HNN simulates these primary currents from the model pyramidal neurons and produces dipole outputs in units of ampere-meters (Am), allowing for direct comparisons between simulated and empirical AEFs. Currents that flow into the cortex (e.g. $\mathrm{N} 100 \mathrm{~m}$ ) correspond to current flow down the pyramidal neuron dendrites in $\mathrm{HNN}$, as detailed further below.

\section{Statistical Analysis}

In order to quantify the differences in the AEF waveform between left and right, as well as between contralateral and ipsilateral hemispheres, we focused on the analysis of the N100m response. Although we do not expect AEF differences to be expressed exclusively in the $\mathrm{N} 100 \mathrm{~m}$, this component is typically the most prominent of the AEF components, and therefore lends itself to quantification. To this end, we conducted a total of four $2 \times 2$ repeated-measures ANOVAs with factors Tone Presentation (contralateral/ipsilateral) and Hemisphere (right/left) to determine differences in N100m amplitude, N100m latency, P50m-N100m slope, and N100m-P200m slope. The N100m trough was defined per participant average as the absolute signal maximum during the time interval from 80 to $120 \mathrm{~ms}$ relative to the auditory stimulus onset. The P50m-N100m, and N100m-P200m slopes were determined by fitting straight lines to each participant's average waveform between 70-90 and 110-130 ms respectively.

We applied the same ANOVAs to the model simulation (see below), by running 10 simulations per modelled condition as a source of variability. While the reduced variability of simulations compared to human MEG data means that these statistics are not directly comparable to those of the empirical data, they nevertheless give insight into whether specific qualitative features, such as differences in slopes or latencies, are reproduced by the model.

\section{Model}

\section{The Human Neocortical Neurosolver (HNN)}

We used the computational neural modeling software Human Neocortical Neurosolver (HNN; Neymotin et al. 2020) to study the neural mechanisms generating the observed source localized, grand-average AEF waveforms. HNN is an open-source software (for tutorials and detailed descriptions of the model, please visit http://hnn.brown.edu), which simulates the primary electrical currents underlying macro-scale EEG and MEG data, based on their biophysical origin by modeling neocortical cellular and circuit-level activity. The model contains multi-compartment pyramidal neurons as well as single-compartment inhibitory interneurons (basket cells) in supragranular layers (layer II/III) and infragranular layers (layer V, see Fig. 1). The morphology of the pyramidal neurons was modelled based on cat visual cortex pyramidal neurons (Bush and Sejnowski 1993), and adjusted in accordance with anatomical findings in the human brain (Geyer et al. 1997; Fischl and Dale 2000; Elston et al. 2001). The intracellular electrical current flow in the long and spatially aligned apical dendrites of the pyramidal neurons across the infragranular and supragranular layers are the main contributors to macroscale primary current dipoles that generate macroscale signals that can be observed on the scalp (Murakami and Okada 2006). The cells are connected with glutamatergic and GABAergic synapses, and each cell's activity is simulated using Hodgkin-Huxley dynamics (Fig. 1a). The primary current dipole is calculated by summing the intracellular current flow across the network of pyramidal neurons (see red and green arrows in Fig. 1c and d). Importantly, the current dipole generated in HNN is expressed in units of ampere-meters (Am), the same units as those estimated by source localization methods of EEG and MEG data, enabling one to one comparison between model and empirical data. For computational tractability, a reduced network size is simulated and a scaling factor is multiplied 
(a)

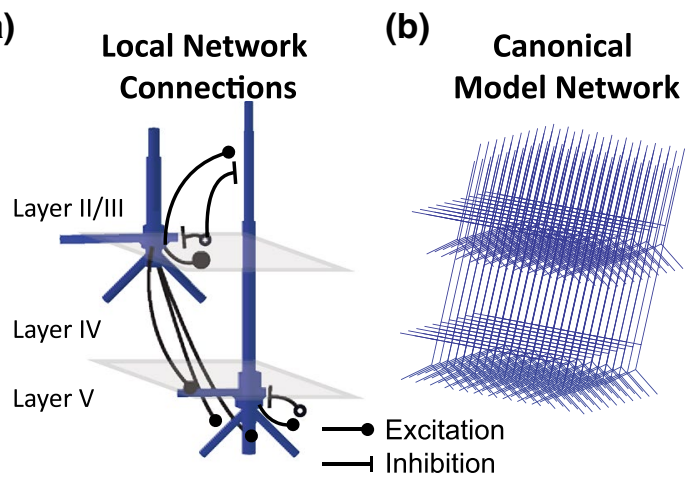

(c)

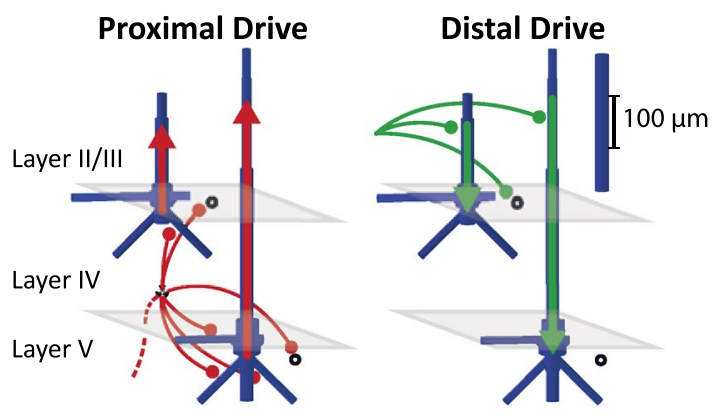

Fig. $1 \mathrm{HNN}$ Model schematics. a Pyramidal neurons in layer II/III and $\mathrm{V}$, and inhibitory fast-spiking basket cells (empty circles). Excitatory and inhibitory synaptic coupling is indicated by black lines with filled circles and bars respectively. Within-layer excitatory-excitatory and inhibitory-inhibitory connections are not shown, but exist for each cell type (see Table S2). b Visualization of the spatial alignment of a network of layer II/III and V pyramidal neurons. c, d The network is activated by proximal/feedforward (c) and distal/feedback (d) inputs which deliver trains of action potentials via canonical pathways

by the aggregate current dipole to estimate the size of the network contributing to the recorded data, as detailed below. To assess the correspondence between averaged evoked responses and modelled dipoles, simulations are smoothed (30 ms Hamming window, see Table S2). There are many more sources of variability in the larger network contributing to the recorded human data, such as spike variability across the network and individual subject differences. We assume high-frequency components created by this variability are more likely to average out in the larger network and multi-subject averages contributing to the recorded AEFs than in model simulations. We therefore apply smoothing to model simulations to allow for a direct comparison between simulation and data (see also Fig. S2). We also simulate $\mathrm{N}=10$ trials per example, each of which has some intrinsic variability, and average across trials.

The baseline network consists of 100 pyramidal neurons per layer (Fig. 1b). A scaling parameter is then applied by multiplying the dipole simulated by the baseline network by a constant. This constant represents the number of neurons contributing to a given recorded signal, assuming the signal represents the summed activity of a larger population of synchronous pyramidal neurons. Since a typical MEG/ EEG response has a magnitude of 10-100nAm, and a single pyramidal neuron contributes approximately $0.2 \mathrm{pAm}$, between 50,000 and 500,000 cells are contributing to a typical macroscale signal, corresponding to a scaling factor of between 250 and 25,000 (Murakami and Okada 2006; Neymotin et al. 2020).

In order to simulate macroscale MEG/EEG signals, the network modelled in HNN is activated through exogenous excitatory synaptic inputs generated by predefined trains of action potentials that contact layer specific post-synaptic targets via two canonical input pathways, which have previously been shown in animal models (Rockland and Pandya 1979; Friedman and Jones 1980; Kulics and Cauller 1986; Cauller and Kulics 1991; Douglas and Martin 2004). We refer to the two types of inputs used here as proximal or feedforward and distal or feedback inputs. Feedforward drives represent signals that reach the cortex from the lemniscal thalamus to granular layers, which then propagate directly to supragranular and infragranular layers where they effectively target the proximal dendrites of the pyramidal neurons (Fig. 1c). Feedback drives represent inputs from non-lemniscal thalamus or cortico-cortical connections that target the distal dendrites in supragranular layers (Fig. 1d). The level of detail included in HNN's model enables simulation of the macroscale current dipoles along with microscale circuit information, including layer specific spiking activity in individual cells. Detailed descriptions of the model underlying HNN can be found in the Supplementary Materials (S1: Supplemental Model Description).

Although the cortical column modelled in HNN was originally generated to account for the anatomy and behavior of primary somatosensory cortex (Jones et al. 2007, 2009), it is based on canonical features shared between different neocortical circuits and hence can be applied to interpret signals in other cortical areas (Neymotin et al. 2020). This is particularly the case for sensory areas, as the relay of sensory information has been shown to follow remarkably stereotypical patterns across modalities (Douglas and Martin 2004; Barbour and Callaway 2008).

Since HNN is a complex model with a large number of parameters, many of which interact, we cannot rule out that some alternative parameter sets could not reproduce similar results (see "Discussion" section). HNN was designed to be a hypothesis development and testing tool. The hypothesis testing here was motivated from our prior studies of sensory evoked responses, which provided the baseline assumptions for the pattern of exogenous proximal and distal drives underlying an evoked response, and prior literature on neural mechanisms underlying hemispheric dominance (see "Introduction" section). While we cannot show that any given model provides a unique solution, it is important to note 
(a)

\section{Left Hemisphere}

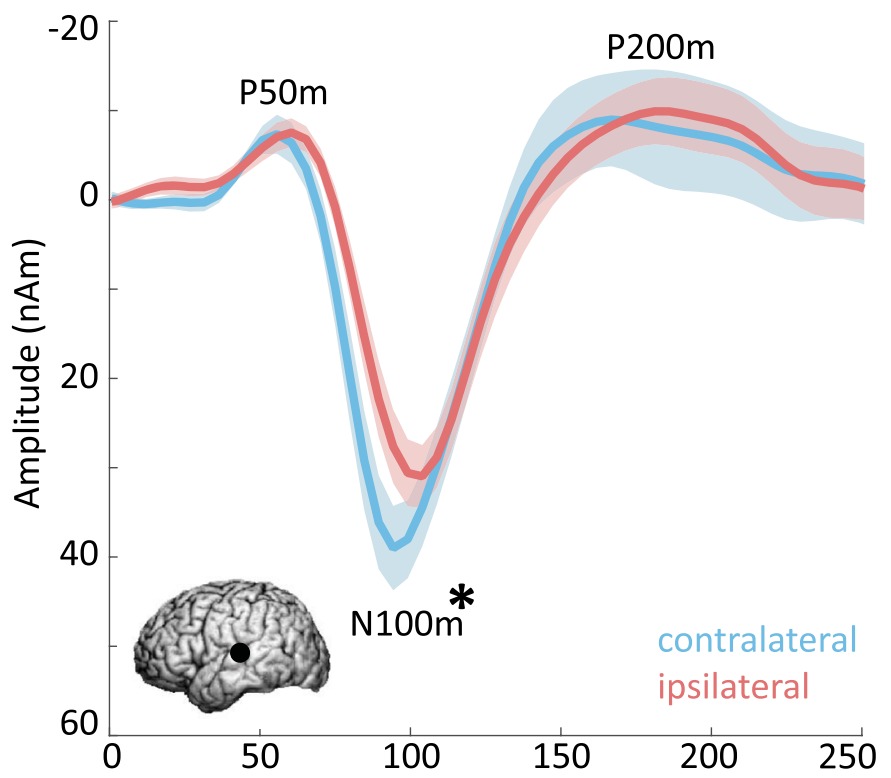

(d)

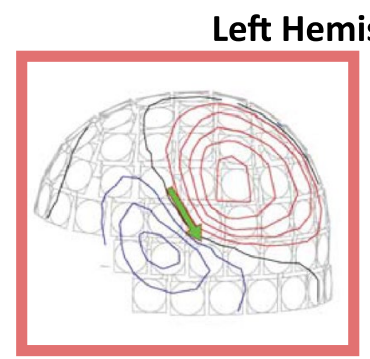

ipsilateral

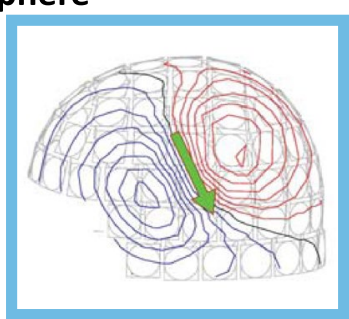

contralateral

(b)

\section{Right Hemisphere}

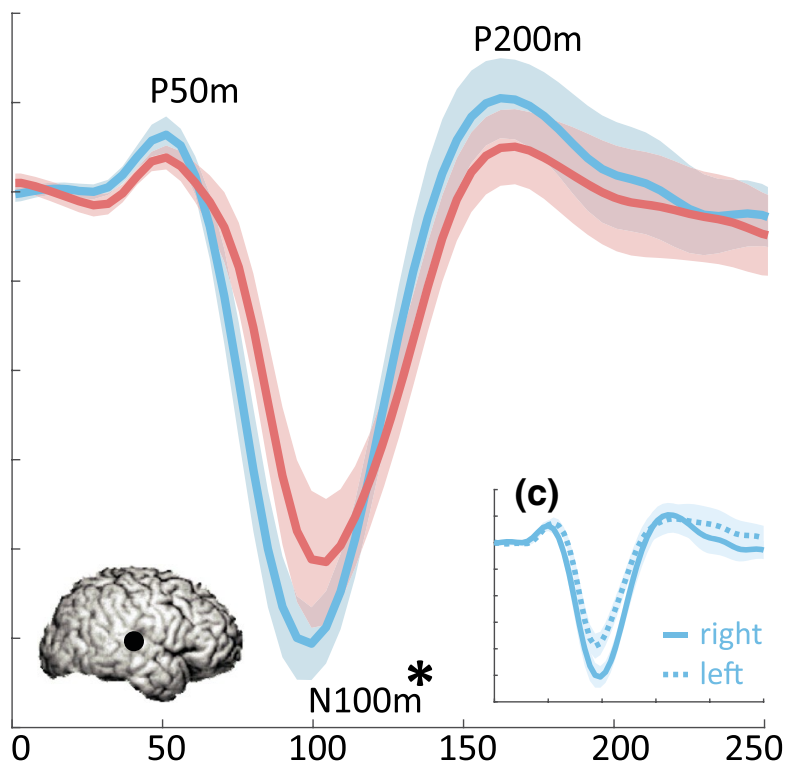

(e)

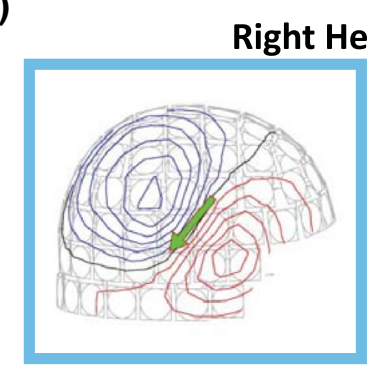

contralateral
Fig. 2 Source-localized grand average AEFs: a, b AEF waveforms in the left (a) and right (b), as well as the contralateral (blue) and ipsilateral (red) hemisphere. Inserts show average source locations. c For ease of comparison, (c) shows left (dotted line, corresponding to blue line in (a) and right (solid line, corresponding to blue line in (b) con- that the dipole shape produced by HNN depends crucially on the underlying assumptions and we can examine the dipole response under alternative hypotheses. To illustrate this, we show models using alternative assumptions for two of our examples (see Fig. 5 and supplement S2, Fig. S1).

\section{Results}

\section{Contralateral and Right Hemisphere AEFs Display More Prominent N100m Peaks}

Source-localized grand average AEFs and their descriptive statistics are displayed in Figs. 2 and 3 respectively. The differences between contralateral and ipsilateral, as well as tralateral AEFs overlaid. Shaded areas indicate SE. *Significant differences in N100m amplitude at $p<0.05$. d, e Magnetic field patterns (at $100 \mathrm{~ms}$ ) of one example subject in the left (d) and right (e), as well as contralateral (blue) and ipsilateral (red) hemispheres

left and right hemispheres were quantified for the N100m component using Tone Presentation $\times$ Hemisphere ANOVAs. As expected based on prior studies (e.g. Peronnet et al. 1974; Wolpaw and Penry 1977; Pantev et al. 1998), we found that $\mathrm{N} 100 \mathrm{~m}$ magnitudes were larger in contralateral compared to ipsilateral $\left(F(1,9)=7.72, p=0.021, \eta^{2}=\right.$ $0.46)$ and right compared to left hemispheres $(F(1,9)=5.71$, $p=0.041, \eta^{2}=0.39$; interaction $\left.p>0.67\right)$. N100m latency differences did not reach significance, but there was a trend towards shorter latencies in contralateral compared to ipsilateral N100ms $\left(F(1,9)=5.08, p=0.051, \eta^{2}=0.17\right.$; all other effects $p>0.21)$. The ascending and descending slopes of the $\mathrm{N} 100 \mathrm{~m}$ trough from the $\mathrm{P} 50 \mathrm{~m}$ to $\mathrm{N} 100 \mathrm{~m}$, and $\mathrm{N} 100 \mathrm{~m}$ to $\mathrm{P} 200 \mathrm{~m}$, were also greater in contralateral compared to ipsilateral AEFs $\left(F_{\mathrm{P} 50 \mathrm{~m}-\mathrm{N} 100 \mathrm{~m}}(1,9)=10.55, p_{\mathrm{P} 50 \mathrm{~m}-\mathrm{N} 100 \mathrm{~m}}=0.01\right.$, 
Fig. 3 Means of empirical and simulated AEFs for each quantified N100m characteristic and each condition. $p$ values are displayed for each ANOVA. Effects demonstrated in both empirical and simulated AEFs are printed in bold. Statistics associated with simulated AEFs are printed in gray as the limited variability in simulations does not allow for direct comparison with tests performed on empirical data. Error bars indicate standard error. ${ }^{*} p<0.05$; $* * * p<0.001$. a N100m amplitude, b N100m latency, c P50N100m slope, d N100m-N200m slope (Color figure online)
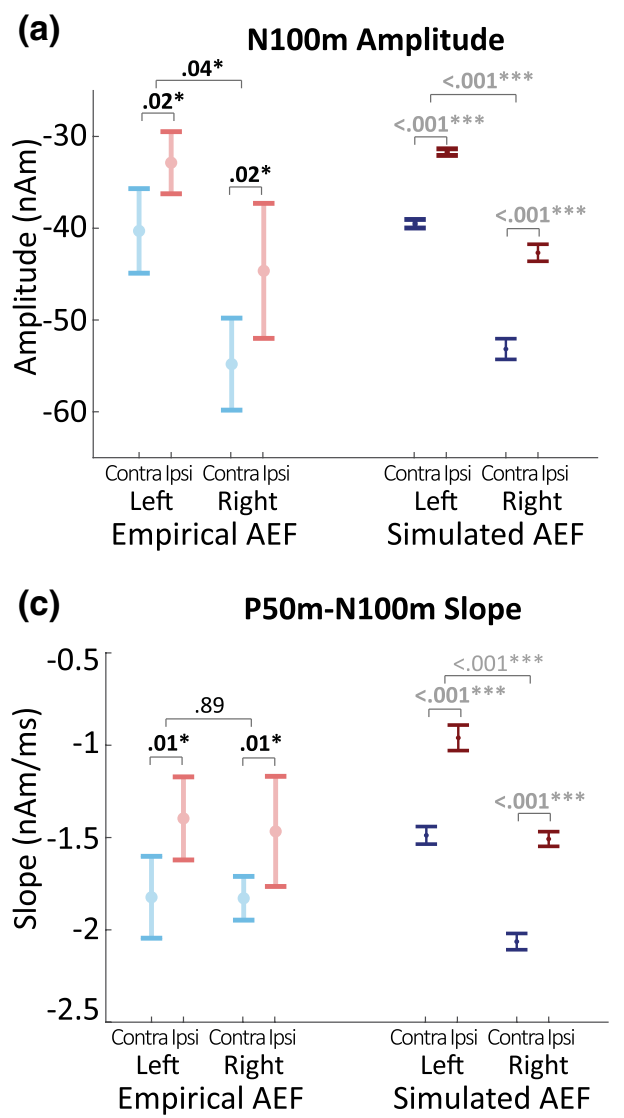
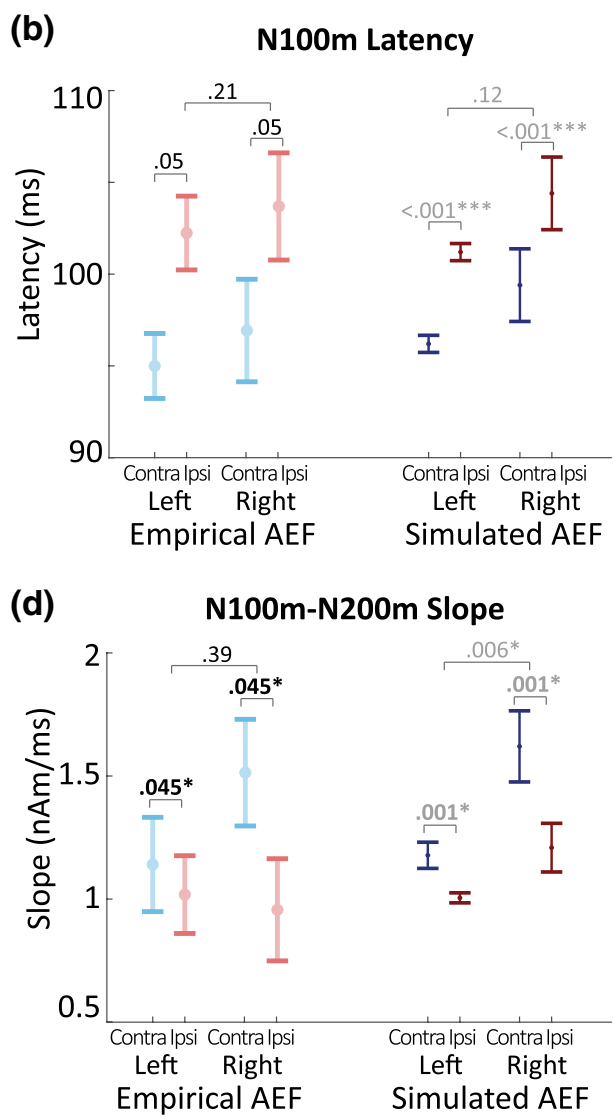

$\eta_{\mathrm{P} 50 \mathrm{~m}-\mathrm{N} 100 \mathrm{~m}}=0.54 ; F_{\mathrm{N} 100 \mathrm{~m}-\mathrm{P} 200 \mathrm{~m}}{ }^{2}(1,9)=5.42, p_{\mathrm{N} 100 \mathrm{~m}-\mathrm{P} 200 \mathrm{~m}}$ $=0.045, \eta_{\mathrm{N} 100 \mathrm{~m}-\mathrm{P} 200 \mathrm{~m}}{ }^{2}=0.38$; all other effects $p>0.092$; Fig. 3).

\section{A Sequence of Feedforward-Feedback-Feedforward Activation of the Cortical Circuit Reproduces AEF in HNN}

The tone evoked AEFs in Fig. 2 exhibit peak timings and polarities that are similar to source localized SEFs evoked by brief perceptual threshold level tactile stimuli observed in our prior studies (e.g. Jones et al. 2007, 2009), where there is an initial small amplitude positive peak (confirmed to correspond to current flow out of cortex), followed by a prominent negative peak (current flow into cortex), followed by a subsequent positive peak. Motivated by this consistency along with the homology of canonical cortical circuitry in sensory areas built into HNN, we applied the input sequence of the existing SEF model distributed with HNN as a starting point for our AEF simulation (namely the 'default.param' parameter file). This sequence consisted of a proximal input, followed by a distal input, followed by a second subsequent proximal input (Jones et al. 2007; Neymotin et al. 2020), and represents a canonical feedforward, feedback, feedforward pattern of activation. This pattern of activation was able to account for rough features of the AEF waveform. To produce a closer fit to the AEF data we began by adjusting only the parameters defining the strength and timing of each input (all input parameters are displayed in Table 1), as well as the scale of the waveform. We first hand-tuned these parameters until there was visually close agreement between model and data, and subsequently used the automated parameter optimization feature in HNN to improve the model fit further. The optimization tool automatically adjusted parameters controlling input timing and strengths to minimize the error (root mean squared error; RMSE) between the simulated and empirical dipole waveforms (Neymotin et al. 2020). This was initially performed for the AEF recorded over the contralateral, right hemisphere. The resulting model simulation (RMSE: 1.0) and its input parameters are displayed in Fig. 4d-f and Table 1, respectively (see Supplementary Materials S2 for alternatives of this model).

Briefly, the input sequence affects the network in the following way: first, a feedforward input reaches the proximal dendrites, as well as inhibitory interneurons of layer II/III and layer $\mathrm{V}$. This input is thought of as feedforward sensory information reaching the auditory cortex via the lemniscal thalamus. It reaches the network around $47 \mathrm{~ms}$ after the onset of the auditory stimulus, strongly driving layer II/III cells (Table 1). This timing is broadly in line with previous 

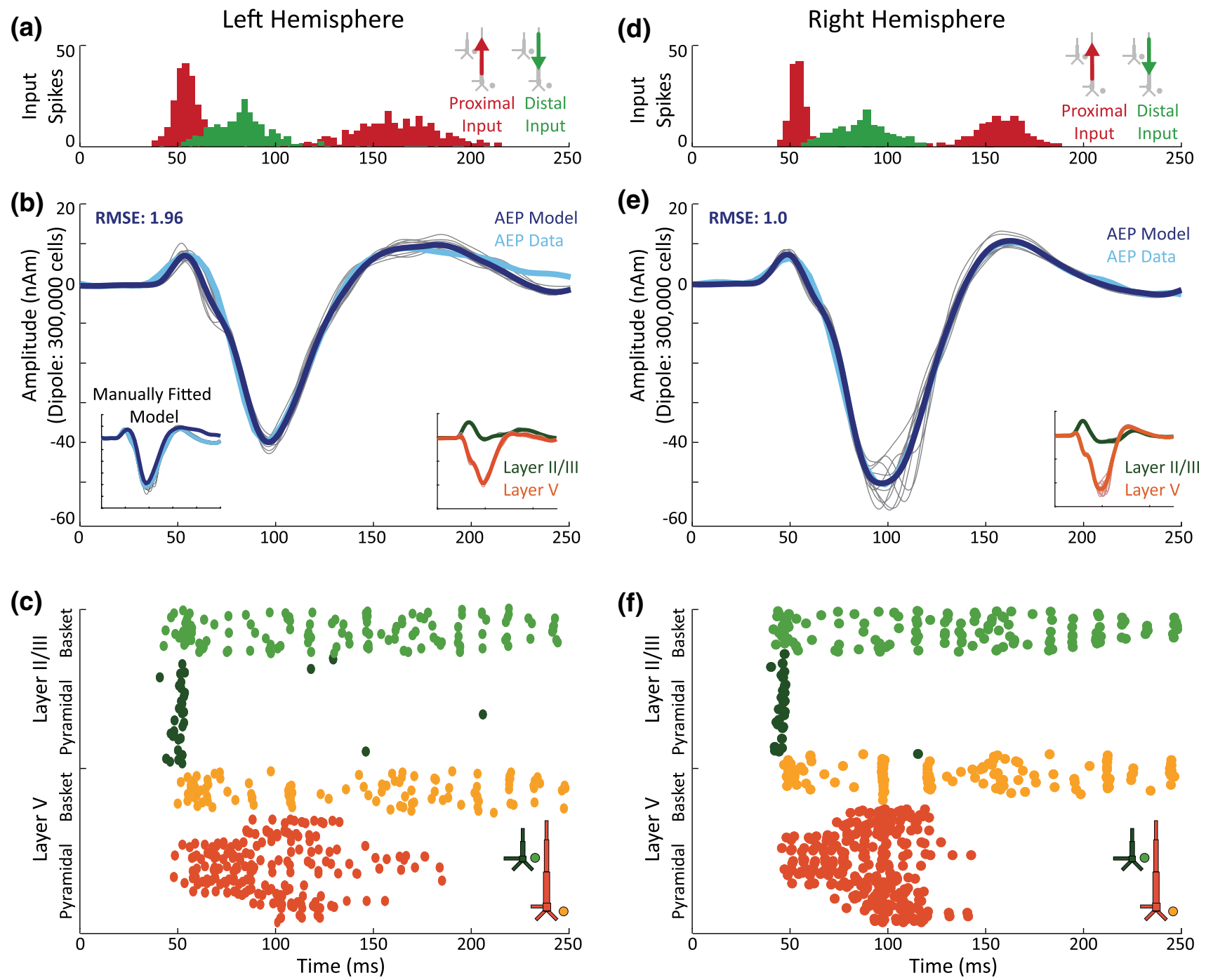

(f)

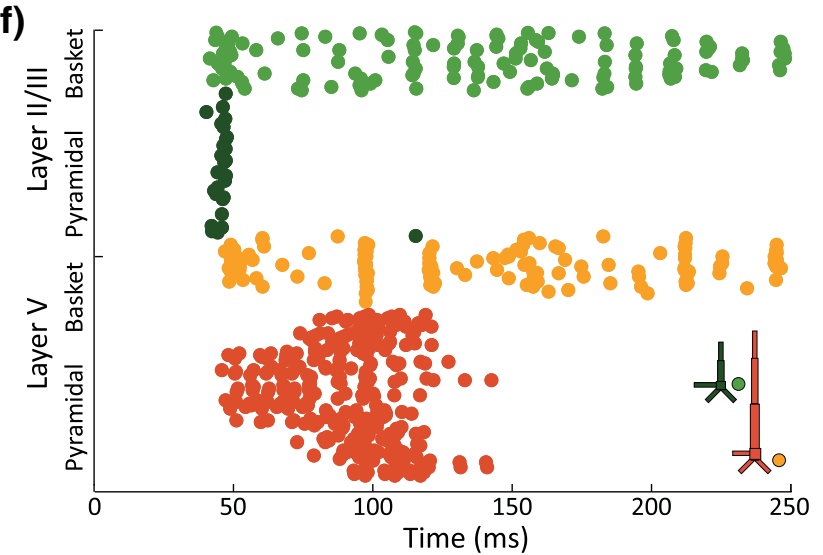

Fig. $4 \mathrm{HNN}$ simulation of the AEF recorded in response to contralateral tone presentation over the right hemisphere (right panels) and over the left hemisphere (left panels): a, d Input sequence: Input spikes are sampled from a Gaussian distribution (mean and sd are defined by input time and sd, see Table 1) on each trial. The resulting temporal profile of the spiking activity arriving into the network is displayed in red (proximal) and green (distal) histograms. A proximal input drives the network, before a distal input and a second proximal input arrive (see Fig. 1c, d for proximal/distal inputs). Corresponding input parameter values are displayed in Table 1. b, e Dipole Simu-

findings, suggesting that AEF components occur after auditory signals reach the cortex at around $50 \mathrm{~ms}$ (Picton et al. 1974). This input causes both pyramidal and basket cells in layer II/III to fire, with pyramidal firing quickly inhibited by the inhibitory basket cells (Fig. 4f). The spiking in the pyramidal neurons creates backpropagation of current flow up the pyramidal apical dendrites towards the surface of the cortex, leading to the P50m peak seen in the AEF (Fig. 4e).

While the effects of the proximal input are ongoing, a feedback input arrives at the distal dendrites at around

lation: mean AEF model (dark blue) as well as 10 individual trial simulations (gray). The empirical AEF (here: contralateral AEF) is displayed in light blue (cf. Fig. 2). Insert at the bottom right shows the dipoles of layer II/III and layer V separately. Left inset in b shows model fit of manually fitted model (with no automatic optimization applied). All dipoles were smoothed using the default settings in HNN (30 ms Hamming window; see Table S2). An unsmoothed equivalent to panel e is displayed in Fig. S2. c, f Simulated spiking activity: spiking associated with the dipole displayed in (b) (one example trial selected) (Color figure online)

$81 \mathrm{~ms}$ (although with a wider temporal distribution, Fig. 4d), strongly activating layer II/III basket and pyramidal cells and layer $\mathrm{V}$ pyramidal cells and inducing the N100m peak. This drive is presumed to represent inputs from either cortico-cortical, or non-lemniscal thalamocortical connections. The distal drive induces several changes in the network dynamics, the net effect of which is a downward deflecting current. Excitatory synaptic inputs on distal apical dendrites of layer II/III and layer V pyramidal neurons push the current flow down (Fig. 1d). At the same 
Table 1 Input parameters used to simulate AEFs in the left and right hemisphere after contralateral tone presentation

\begin{tabular}{|c|c|c|c|c|c|c|c|}
\hline \multirow[t]{2}{*}{ Parameter } & & \multicolumn{3}{|c|}{ Left hemisphere model } & \multicolumn{3}{|c|}{ Right hemisphere } \\
\hline & & Proximal & Distal & Proximal & Proximal & Distal & Proximal \\
\hline Input time & & 54.898 & 82.99 & 161.307 & 47.355 & 81.09 & 150.826 \\
\hline SD & & 5.401 & 13.208 & 19.844 & 2.963 & 12.26 & 11.061 \\
\hline \multirow{2}{*}{$\begin{array}{l}\text { Layer II/III } \\
\text { Pyramidal }\end{array}$} & AMPA & 0.991 & 0.607 & 0.854 & 0.659 & 0.607 & 0.346 \\
\hline & NMDA & 1.714 & 0.242 & 0.067 & 0.535 & 0.243 & 0.027 \\
\hline \multirow[t]{2}{*}{$\begin{array}{l}\text { Layer II/III } \\
\text { Basket }\end{array}$} & AMPA & 0.997 & 0.624 & 0.758 & 0.997 & 0.523 & 0.995 \\
\hline & NMDA & 0.984 & 0.953 & 0.851 & 0.987 & 0.959 & 0.994 \\
\hline \multirow[t]{2}{*}{$\begin{array}{l}\text { Layer V } \\
\text { Pyramidal }\end{array}$} & AMPA & 0.004 & 0.258 & 0.012 & 0.004 & 0.964 & 0.005 \\
\hline & NMDA & 0.01 & 0.157 & 0.004 & 0.009 & 0.158 & 0.006 \\
\hline \multirow[t]{2}{*}{$\begin{array}{l}\text { Layer V } \\
\text { Basket }\end{array}$} & AMPA & 0.615 & & 0.98 & 0.427 & & 0.984 \\
\hline & NMDA & 0.062 & & 0.902 & 0.037 & & 0.998 \\
\hline
\end{tabular}

The corresponding simulations are displayed in Fig. 4. Parameters which were identified as most relevant to account for the difference between left and right hemispheres are printed in bold. AMPA and NMDA synaptic weights (i.e. maximal conductances) are displayed in units of $\mu \mathrm{S}$, while mean and standard deviation of the input times are displayed in ms. Note that distal inputs do not project onto layer $\mathrm{V}$ basket cells (see Fig. 1). Parameter values rounded to three decimal places. All other parameters are displayed in Table S2 time, somatic inhibition mediated by activation of basket cells in both layers (note, the layer $\mathrm{V}$ baskets cells are driven by the layer II/III excitatory connections to layer V, Fig. 1a) pulls current further down the pyramidal neuron dendrites. In addition, activation of calcium dynamics, triggered by layer $\mathrm{V}$ pyramidal excitation pulls current down towards infragranular layers. The distal drive also generates spiking activity in the layer $\mathrm{V}$ pyramidal neurons, which leads to backpropagation, i.e. current flow in the opposite direction up the dendrites. However, the size of this effect is smaller and not visible in averaged and smoothed data (see Fig. S2 and "Methods" section) and the overall effect is current flow down the dendrites, leading to a large N100m trough (Fig. 4e).

Lastly, as the spiking activity in the network would begin to relax back to baseline after the distal drive, a second proximal input reaches the network at around $151 \mathrm{~ms}$, driving primarily layer II/III and layer V basket cells, and some pyramidal cells, causing continuous basket firing, with burst-like behavior occurring at around $200 \mathrm{~ms}$ (Fig. 4f). This activation of postsynaptic excitatory synapses on the basal dendrites of the pyramidal neurons again pushes current flow towards the surface of the cortex. Additionally, the layer II/III basket cells inhibit the distal dendrites of the layer V pyramidal neurons (Fig. 1a), helping to pull current flow up the dendrites toward the cortical surface. Together these effects create the $\mathrm{P} 200 \mathrm{~m}$ peak in the AEF.

Dividing the net current dipole into its layer specific components shows that the shape of the AEF simulation is largely driven by responses in layer V, while layer II/III responses contribute only to the earliest component (see inserts in Fig. 4e, and Fig. S2b).

We also calculated the simulated firing rates of each neuronal population by averaging the number of action 
potentials over trials and cells. We found the highest firing rates in basket cells (layer $\mathrm{V}=13.97$ spikes/s; layer II/ III = 13.46 spikes/s), followed by layer $\mathrm{V}$ pyramidal neurons (10.60 spikes/s), and low firing rates in layer II/III pyramidal neurons (1.85 spikes/s, cf. Fig. 4f). Although firing rates were not considered as part of the model fitting procedures, these firing rates appear to be within physiological ranges (Wallace and Palmer 2008; Atencio and Schreiner 2010), further supporting the model-derived predictions.

\section{HNN Predicts Smaller Left Hemisphere AEF N100m Peak can be Generated by Decreased Feedback Drive}

With the AEF recorded from the right hemisphere (for contralateral tone presentation) simulated, we set out to model the AEF recorded over the left hemisphere. Since we did not have any clear hypotheses predicting the differences between left and right hemispheres based on prior literature, we used model derived knowledge of how the inputs to the network influence the timing and magnitude of the peaks to examine how differences in the right and left hemispheres could emerge. Using right AEF as a starting point, we began by tuning the input parameters to fit the left AEF, using both hand-tuning and automatic optimization. The targeted differences we aimed to create were the significantly smaller N100m amplitude (Figs. 2c and 3). By hand tuning each input parameter, we found that modulation of only one input parameter was able to account fairly well for the qualitative differences in the right and left waveforms. Specifically, we found that by decreasing the AMPA mediated excitation of layer $\mathrm{V}$ pyramidal cells by the distal drive (see Table 1 and Table S2), and keeping all other parameters fixed between hemispheres, we were able to reproduce the AEF waveform associated with the left hemisphere (Table 1). Since distal layer $\mathrm{V}$ pyramidal excitation pushes currents down the dendrites, creating the $\mathrm{N} 100 \mathrm{~m}$ trough, decreasing the excitatory inputs to these cells leads to a smaller N100m magnitude as seen in left hemisphere AEFs. We refer to the model in which only this parameter was changed to account for left AEFs as the 'Manually Fitted Model', and used automated parameter optimization to further improve the model fit. While further small parameters changes were needed to optimize the model fit (Fig. 4b), fitting the model with only this one parameter was sufficient to reproduce the targeted differences (see "Manually Fitted Model" in Fig. 4b). The optimized model (Fig. 4b, large panel) was used for all further analyses. The resulting dipole simulations displayed the same N100m peak amplitude difference between left and right hemispheres as the empirical AEF $(p<0.001)$.

While our initial testing of the hypothesis that strength of the exogenous distal inputs would be essential to defining the amplitude of the AEF peaks confirmed that alteration in this parameter can reproduce the observed differences, we cannot rule out that alternative mechanisms could reproduce similar results. To address this issue, we also tested two alternative models that could logically account for N100m amplitude differences through alteration of local network features, rather than inputs into the network. Since simulations show that both somatic inhibition and layer $\mathrm{V}$ calcium activity can lead to current flow down the dendrite (i.e. larger N100m amplitudes), we decreased the parameter values representing each one at a time to test if these changes could also lead to less current flow down the dendrite and therefore smaller N100m amplitudes as seen in left hemisphere AEFs. We found that by decreasing the parameters controlling the synaptic weights of all local network connections targeting inhibitory cells, as shown in Fig. 1a, by a factor of 10 from the right hemisphere AEF parameters, the model was indeed able to approximate the decreased N100m amplitudes of left hemisphere AEFs (Fig. 5a). Specifically, we decreased five parameters, representing the conductance of the synaptic connections targeting layer $\mathrm{V}$ basket cells from layer II/III and layer $\mathrm{V}$ pyramidal cells, and layer $\mathrm{V}$ basket cells, as well as connections targeting layer II/III basket cells from layer II/III pyramidal and basket cells. However, this manipulation also impacted the subsequent $\mathrm{P} 200 \mathrm{~m}$ peak, which was significantly larger when the local connections to the inhibitory neurons were decreased due to increased pyramidal neuron firing (data not shown), and hence a larger RMSE between the model and recorded data were observed; $\mathrm{RMSE}=7.84$ compared to RMSE = 1.96 in Fig. 4. Similarly, we found that the $\mathrm{N} 100 \mathrm{~m}$ amplitude decrease could also be reproduced by a simulation in which the calcium channel density in the soma and along the dendrites of the layer $\mathrm{V}$ pyramidal neurons (Table S2), was decreased by approximately $94 \%$ from the right hemisphere AEF parameters (Fig. 5b). In this case, the subsequent $\mathrm{P} 200 \mathrm{~m}$ amplitude was smaller than recorded data due to decreased pyramidal neuron firing (data not shown), and hence the RMSE between the model and recorded data was again larger than before; $\mathrm{RMSE}=6.57$ compared to $\mathrm{RMSE}=1.96$. These results suggest that initial manipulations of the strength of the distal input as in Fig. 4; Table 1, was more effective at reproducing multiple features of the empirical data and we therefore used the initial left AEF model for all following analyses.

\section{Differences in the Feedforward and Feedback Input Latencies, and Network Size, Can Reproduce Contralateral/Ipsilateral AEF Differences in HNN}

After establishing mechanisms that can reproduce the contralateral AEFs in both the left and right hemisphere, we next tested the specific hypotheses based on prior literature that differences between contralateral and ipsilateral AEFs emerge 


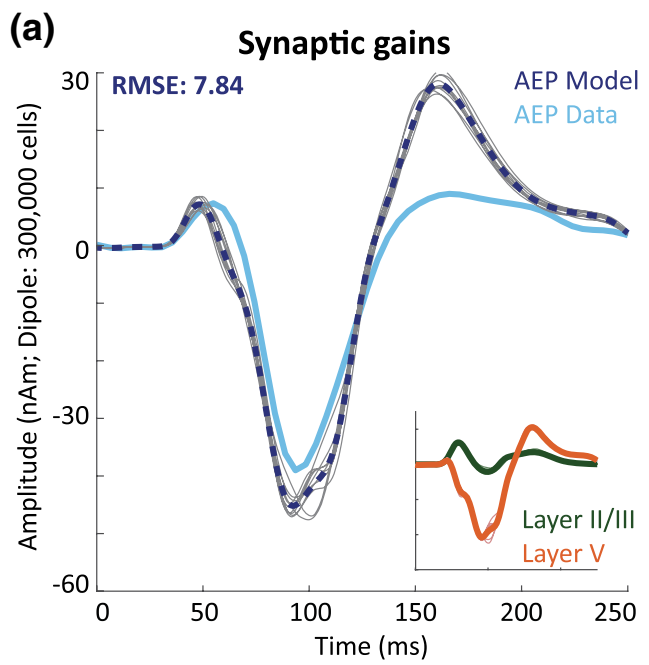

Fig. 5 Alternative simulations for left hemisphere AEFs: left hemisphere AEFs (contralateral) are displayed in light blue, while model simulations are displayed in dark blue dotted lines. Individual simulations are displayed in gray. Inserts show the dipoles associated with layer II/III and layer V separately. The fit provided by the alternative simulations was noticeably worse than that of the initial model,

from differences in the size of the active network (Rosenzweig 1951; Gross et al. 1967), and alterations in the timing of the exogenous input to the network (Tunturi 1946; Kimura 1961). The differences that we targeted to reproduce in the model were the decreased $\mathrm{N} 100 \mathrm{~m}$ amplitude, and smaller P50m-N100m and N100m-P200m slopes as observed in the empirical data (Figs. 2 and 3). We specifically hypothesized fewer cells contributed to the ipsilateral compared to the contralateral AEFs, and thus we first hand-tuned the scaling parameter in the model that provides an estimate of the number of neurons contributing to the observed data (see Methods), decreasing from a value of 1500 in the contralateral AEF model to 1200 for the ipsilateral AEF simulation. This change was able to account well for the decreased N100m magnitude in the ipsilateral versus contralateral AEF, and also improved the model fit to the ipsilateral AEF around the P50m and $\mathrm{P} 200 \mathrm{~m}$ components, compared to the contralateral simulation. This change in scale also accounted for the decreased P50m-N100m and N100m-P200m slopes associated with ipsilateral AEFs (Fig. 3). As such, the model results predict that a smaller network with on the order of 240,000 pyramidal neurons $(1200 \times 200 \mathrm{PN}$ per layer) generated the ipsilateral response compared to $300,000(1500 \times 200 \mathrm{PN}$ per layer) for the contralateral response (Fig. 6). Since previous findings further suggest a difference in latency between contralateral and ipsilateral AEFs (Pantev et al. 1986, 1998), in a second step, we hand tuned the mean latency of the inputs into the network and found that the model fit to the data was improved when all three inputs were delayed by $5 \mathrm{~ms}$. Since the latencies of all inputs were adjusted equally, this manipulation did

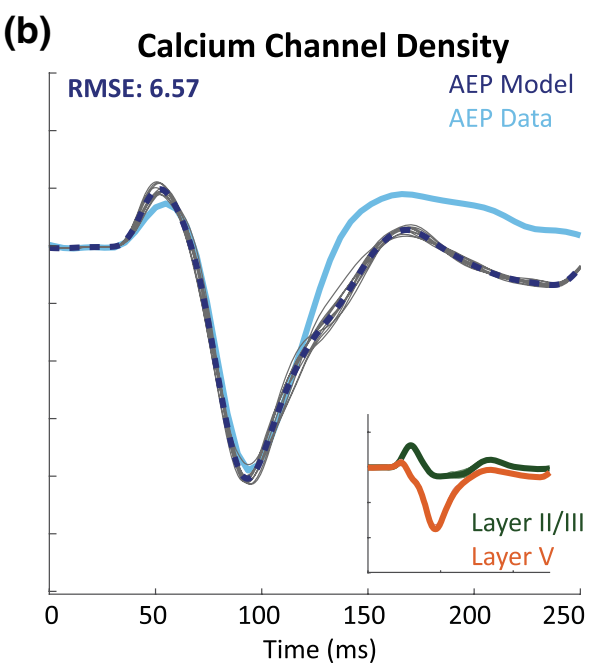

which only adjusted input parameters (cf. Fig. 4b). a Synaptic gains were decreased in all connections targeting inhibitory interneurons. b Layer V pyramidal calcium channel densities were decreased. All other parameters were equal to the model of the right hemisphere AEF (see Fig. 4d-f; Table 1) (Color figure online)

not change the shape of the dipole, but shifted it along the time axis. Although latency differences between contralateral and ipsilateral AEFs were not statistically significant in our data, N100m latencies were slightly but consistently larger in ipsilateral AEFs (Fig. 3). This trend was captured well by simulations incorporating this $5 \mathrm{~ms}$ shift. The resulting AEF simulations are displayed in Fig. 6.

Both scaling and time parameters were hand-tuned for the difference between contralateral and ipsilateral AEFs to account first for only the right hemisphere. The same modulations, i.e. decreasing the scale by 300 and increasing the input times by $5 \mathrm{~ms}$, were then applied to the model of the left hemisphere AEF without further fitting steps.

By following these two steps, the model provided a good fit to the ipsilateral AEFs of both hemispheres (RMSE right: 2.15, RMSE left: 2.19), displaying the same N100m amplitude and slope differences as the empirical data $(p<0.006$, Fig. 3), without further fitting or optimization steps, confirming the hypothesis that differences in network size and timing of the exogenous inputs can account for waveform shape differences between contralateral and ipsilateral AEFs.

\section{Discussion}

Auditory evoked fields are a commonly used measurement in human studies of healthy cognitive processing and as biomarkers of neuropathology, yet the biophysical mechanisms underlying these scalp-recorded responses are not yet fully understood. While human AEF studies and laminar 


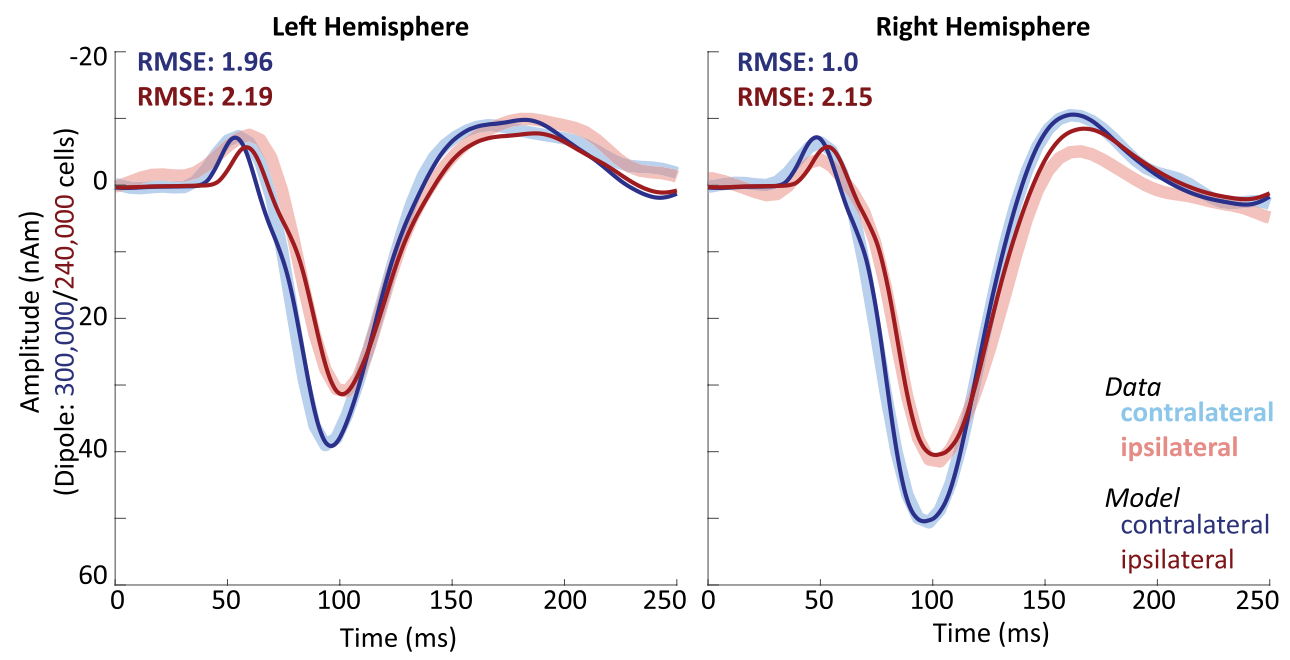

Fig. $6 \mathrm{HNN}$ simulation of the contralateral dominance effect for the right hemisphere (right panel) and the left hemisphere (left panel) AEF. Average simulations (based on 10 individual trial simulations) of contralateral AEFs (dark blue, cf. Fig. 4) and ipsilateral (dark red) AEFs. Ipsilateral responses were generated by decreasing the model

recordings of the auditory cortex in animal models often proceed in relative isolation, here, we connected the information of the circuitry architecture, provided by animal models, with human MEG recordings using biophysically principled computational neural modeling. We used the Human Neocortical Neurosolver (HNN), a software whose foundation is a model of a canonical neocortical circuit, which allows users to define inputs to activate the network and simulates the primary electrical currents underlying scalp-recorded fields or potentials (Jones et al. 2007; Neymotin et al. 2020), to develop and test predictions on the neural mechanisms underlying two well-established characteristics in the AEF response, namely the lateralization effect in which responses recorded over the right hemisphere display stronger magnitudes in response to simple auditory stimuli than those recorded over the left hemisphere (Hine and Debener 2007; Howard and Poeppel 2009; Kimura 2011), as well as the phenomenon that AEFs in response to contralaterally presented tones show larger, and often faster, responses than ipsilaterally presented tones (Tunturi 1946; Rosenzweig 1951; Kimura 1961; Pantev et al. 1998).

We were able to model the AEF waveform elicited by a brief monaural contralateral tone presentation using the same input sequence that has previously been shown to simulate somatosensory evoked responses (Jones et al. 2009), with parameters tuned to match the AEF data. According to this sequence, auditory responses can be accounted for by an initial excitatory thalamocortical feedforward drive to layer II/III and V, via layer IV inducing the $\mathrm{P} 50 \mathrm{~m}$, followed by a cortico-cortical or non-lemniscal thalamic feedback input to supragranular layers inducing scaling factor, representing the number of cells contributing to the signal, and increasing the input latencies, as compared to the contralateral simulations. Empirical AEFs are shown in light colors (cf. Fig. 2) to indicate model fit (Color figure online)

the $\mathrm{N} 100 \mathrm{~m}$, and a subsequent second feedforward input inducing the $\mathrm{P} 200 \mathrm{~m}$. This finding is in line with evidence suggesting that all sensory areas share the same basic structure as well as many defining features, such as the basic laminar organization and activation patterns (Douglas and Martin 2004). Specifically, the initial activation of cortical circuits by the thalamus, which follows a layer IV, layer II/III, layer V information processing sequence, a simplified version of which is recreated here and generates the P50m using HNN, has been shown to be similar across sensory modalities (Douglas and Martin 2004; Barbour and Callaway 2008; Atencio and Schreiner 2010).

It is important to note that the connectivity within the auditory cortex is not as well understood as in other sensory regions, and that focusing solely on similarities between cortical regions is likely an oversimplified description of auditory cortical activation. There are known differences between auditory cortex and other sensory regions, as for example, laminar differences are less prominent than in visual or somatosensory regions, and there is some evidence to suggest that the functional role of auditory layer IV may differ from those in other sensory regions (Linden and Schreiner 2003; Barbour and Callaway 2008; Ji et al. 2016; Oviedo 2017). However, we show that a manually fitted neural model of a cortical column with canonical layer specific input patterns is able to account for scalp-recorded auditory responses, supporting the notion that the basic structure and activation patterns are similar across sensory regions, and providing means to approach the biophysical basis of well-known characteristics and effects reported in human AEF research. 
Further, we found that simulations associated with AEFs recorded over left and right hemispheres were remarkably similar, not only in the input sequence, but also in firing rates and dipole waveforms (compare Fig. 4a-c and d-f). Interestingly, the only parameter necessary to account for qualitative differences in the waveform shape was the strength of the distal input into layer $\mathrm{V}$ pyramidal neurons. In other words, a $100 \mathrm{~ms}$ distal feedback input drives layer $\mathrm{V}$ pyramidal neurons more strongly in right than in left hemisphere AEFs (note that since parameter optimization was performed all input parameters differed somewhat between left and right hemisphere models). These findings are in general agreement with research demonstrating functional asymmetries between left and right hemispheres. For example, right hemisphere dominance has been shown especially in processing of pure tones, although the hemispheric balance seems to depend on the nature of the stimulus, as well as context (Peronnet et al. 1974; Wolpaw and Penry 1977; Schönwiesner et al. 2007; Howard and Poeppel 2009). It could be speculated that the relative specialization of the right hemisphere to process pure tones is associated with optimized connectivity, leading to stronger inputs. It would be interesting, in the future, to explore whether the diminished rightward asymmetry, or leftward asymmetry to speech sounds could be explained by similar underlying dynamics. However, the functional lateralization of auditory processing continues to be debated (e.g. Boemio et al. 2005), and its relation to biophysical mechanisms is unknown. Additionally, our interpretations are model-derived predictions, since we did not have clear pre-defined hypotheses regarding the mechanisms underlying the differences in left and right AEFs.

HNN is designed to be a hypothesis development and testing tool, however in its current form not all possible hypotheses can be explored. For example, macroanatomical differences not captured in HNN between left and right hemispheres could contribute to, if not fully account for, waveform differences observed in AEFs (Galaburda et al. 1978; Rademacher et al. 1993; Penhune et al. 1996; Howard and Poeppel 2009). In fact, Shaw et al. (2013) argued that what may appear as a functional asymmetry in auditory processing is, at least partially, caused by structural asymmetries, as increased cortical folding in the left auditory regions results in increased MEG/EEG signal cancelation. Here, we do not account for these anatomical differences since HNN has been developed to account for the microanatomical principles underlying electrophysiological recordings, and does not include cortical folding in its parameters. As such, we cannot rule out the possibility that the parameter differences between left and right hemisphere AEFs we found in our model are simply compensating for the effect of structural differences, and do not accurately reflect underlying functional mechanisms. However, we found that alternative model adjustments which manipulated local network features, instead of external input characteristics to account for hemispheric AEF differences, led to a poor model fit, providing some support for our model. Ultimately, the predictions made with $\mathrm{HNN}$ can guide targeted experiments to validate or negate model results, and new data informs HNN expansion and improvement.

We also simulated the contralateral dominance effect, i.e. the larger N100m amplitude in the AEF waveform associated with contralateral compared to ipsilateral tone presentation. In the AEF literature, the mechanistic differences proposed to underlie the finding that increased AEF amplitudes are recorded in hemispheres contralateral to the auditory stimulus, are that auditory pathways that cross over are stronger and/or more numerous than ipsilateral ones (Tunturi 1946; Rosenzweig 1951; Kimura 1961), and that the cortical region activated by the auditory stimulus is larger in the contralateral hemispheres (Rosenzweig 1951; Gross et al. 1967). We tested these predictions using HNN. We expected the strength of a pathway to correlate primarily with the speed of the relay of information, which corresponds to the input time parameters in HNN. The notion of differences in the size of the cortical area can be accounted for by HNN's scaling parameter, which gives an estimate of the number of cells contributing to a given dipole. We were able to recreate the differences between contralateral and ipsilateral AEFs, solely by changing the time and scaling parameters, supporting the hypotheses that these manipulations based on animal research can indeed account for the differences in the human signal.

These simple manipulations led to greatly improved model fits to ipsilateral data, in both the left and the right hemisphere. The ipsilateral model fits the ipsilateral AEF less well than the contralateral model fits its corresponding data which is noticeable particularly in the $\mathrm{P} 200 \mathrm{~m}$ peak fit in the right hemisphere. However, note that the contralateral models were established by hand-tuning and then optimizing all 28 input parameters (Table 1), while the ipsilateral model was generated by adjusting only four parameters, three of which by a fixed value. Importantly, the four parameters, as well as the directions in which to adjust them (i.e. increasing the time of each of three inputs by a fixed amount and decreasing the network scale), were chosen based on predictions from the literature, not on the patterns identified in our data, and no further steps were taken to fit the model simulations to the empirical AEFs. In view of this procedure, we consider the ipsilateral models to reproduce the corresponding AEFs remarkably well.

We found that, compared to ipsilateral AEFs, contralateral AEFs can be simulated by increasing the scaling factor by $20 \%$ and decreasing the time of each input by $5 \mathrm{~ms}$. The difference of $20 \%$ found here is also approximately in line with predictions made by Rosenzweig (1951) who suggested that the size of ipsilateral presentations is around 
$25 \%$ smaller than contralateral representations. Although in our data, we were not able to demonstrate clear latency differences between contralateral and ipsilateral AEFs, we found that a difference of $5 \mathrm{~ms}$ provided improved model fits. This small difference is in line with previous studies which have reported contralateral responses to occur between 4 and $14 \mathrm{~ms}$ faster than ipsilateral ones (Mononen and Seitz 1977; Mäkelä et al. 1994; Pantev et al. 1998). Since this is a fairly small difference in latency, it may overlap with individually differing factors that influence AEF measures at these latencies, and may not be easily demonstrated. We speculate that this may explain why other studies, like our own, have not been able to confirm latency differences between ipsilateral and contralateral AEFs (Yoshiura et al. 1994). Overall, we found that ipsilateral AEFs, compared to contralateral ones, can be simulated by delaying the activation of the cortical network and decreasing the size of the network, supporting previous hypotheses predicting that contralateral pathways are faster and activate larger regions of the cortex.

It is important to note that, since HNN is a large-scale model with thousands of differential equations and parameters, we cannot claim that any given waveform has a unique way in which to model it. With large numbers of parameters, many of which interact and trade off, HNN is primarily a hypothesis testing tool as it is not feasible to test the entire parameter space. However, a large proportion of the model, including cell morphologies and physiologies, and local connectivity patterns were fixed based on a large body of animal models, introducing biologically realistic constraints (Jones et al. 2007; Neymotin et al. 2020). Further, we drastically limited the number of parameters free to vary, by making the assumption that many basic features of the network are preserved across sensory areas, which allowed us to fix the vast majority of parameters to values previously established for somatosensory response models (Jones et al. 2007, 2009). Based on the same assumption, we also fixed the input sequence which activates the network, but not the timing and strength of the inputs. This resulted in a model with 28 input parameters and one scaling parameter, while all other parameters were fixed (seven additional parameters, representing calcium activity and inhibitory connection strength, were adjusted to test alternative models, but were not used in the final models). In the case of interpreting the difference between left and right hemispheres, we attempted to narrow down the parameter space of interest by identifying which changes made the largest contribution to the difference in waveform shape. However, we cannot rule out the possibility that other parameter values could account for the finding in a different way. In the case of contralateral compared to ipsilateral AEFs on the other hand, we had clear hypotheses predicting not only the parameters of interest, but also the direction of their adjustment, enabling much clearer conclusions.

Additionally, it is important to note that HNN is designed to bridge the gap between human macro-scale data and circuit-level findings from animal models. Many of the circuit features built into HNN, including those regulating input drives into sensory cortices, were based on animal studies in rodents and non-human primates and have not been directly demonstrated in humans (see Neymotin et al. 2020; Jones et al. 2007, 2009). Although many basic structures and mechanisms seem to be shared across cortical regions and species (Douglas and Martin 2004), and HNN has been able to account for a variety of human signals (Jones et al. 2009; Sliva et al. 2018; Neymotin et al. 2020), including those with remarkable homology between humans, rodents and non-human primates (Sherman et al. 2016; Shin et al. 2017) we cannot rule out the possibility that some cell- or circuit-level dynamics which are specific to humans are misrepresented in HNN.

Overall, we argue that biophysical modelling of human macro-scale brain signals, using models such as HNN, is an important step to bridge the gap between human cognitive neuroscience, and cell- and circuit-level insights provided by animal models. While we aimed to characterize commonly observed phenomena on a biophysical basis, future research may apply similar approaches to study similar scalp potentials, providing insights into mechanistic differences between developmental stages or clinical populations. Future research may also build on the current capabilities of HNN and incorporate sensitivity analyses to allow for an improved interpretation of parameter values.

In summary, we found that HNN, a model of a canonical neocortical circuit, can be used to simulate human AEFs by activating the network using an input sequence similar to one used to model other sensory responses, supporting the notion that the basic structure and activation patterns are preserved across sensory regions. We show that AEF differences between left and right, as well as contralateral and ipsilateral hemispheres, can be simulated by adjusting a small number of parameters representing network scale and input characteristics. Our simulations establish a connection between scalp-recorded correlates of auditory processing and network-level insights from animal models, providing a first step to understand the mechanisms underlying this cognitively and clinically relevant biomarker.

Supplementary Information The online version contains supplementary material available at https://doi.org/10.1007/s10548-021-00838-0.

Author Contributions SRJ and TP devised the project. TP provided the data. CK carried out the simulations. SRJ supervised the modelling work. SRJ, TP and CK contributed to the interpretation of the 
results. CK drafted the manuscript, which all authors critically revised and approved.

Funding This study was supported by National Institutes of Health (Grant Nos. NIBIB RO1 EB022889, NIMH RO1 MH106174).

Data Availability Averaged data, simulations, and parameter files are available on http://github.com/kohl-carmen/HNN-AEF.

Code Availability HNN is available at http://hnn.brown.edu and http:// github.com/jonescompneurolab/hnn.

\section{Declarations}

Conflict of interest The authors declare that no competing interests exist.

Open Access This article is licensed under a Creative Commons Attribution 4.0 International License, which permits use, sharing, adaptation, distribution and reproduction in any medium or format, as long as you give appropriate credit to the original author(s) and the source, provide a link to the Creative Commons licence, and indicate if changes were made. The images or other third party material in this article are included in the article's Creative Commons licence, unless indicated otherwise in a credit line to the material. If material is not included in the article's Creative Commons licence and your intended use is not permitted by statutory regulation or exceeds the permitted use, you will need to obtain permission directly from the copyright holder. To view a copy of this licence, visit http://creativecommons.org/licenses/by/4.0/.

\section{References}

Andreassi JL, DeSimone JJ, Friend MA, Grota PA (1975) Hemispheric amplitude asymmetries in the auditory evoked notential with monaural and binaural stimulation. Physiol Psychol 3:316-316. https:// doi.org/10.3758/BF03337533

Atencio CA, Schreiner CE (2010) Columnar connectivity and laminar processing in cat primary auditory cortex. PLoS ONE. https://doi. org/10.1371/journal.pone.0009521

Barbour DL, Callaway EM (2008) Excitatory local connections of superficial neurons in rat auditory cortex. J Neurosci 28:1117411185. https://doi.org/10.1523/JNEUROSCI.2093-08.2008

Boemio A, Fromm S, Braun A, Poeppel D (2005) Hierarchical and asymmetric temporal sensitivity in human auditory cortices. Nat Neurosci 8:389-395. https://doi.org/10.1038/nn1409

Bush PC, Sejnowski TJ (1993) Reduced compartmental models of neocortical pyramidal cells. J Neurosci Methods 46:159-166. https:// doi.org/10.1016/0165-0270(93)90151-G

Cauller LJ, Kulics AT (1991) The neural basis of the behaviorally relevant N1 component of the somatosensory-evoked potential in SI cortex of awake monkeys: evidence that backward cortical projections signal conscious touch sensation. Exp Brain Res 84:607-619. https://doi.org/10.1007/BF00230973

Devlin JT, Raley J, Tunbridge E et al (2003) Functional asymmetry for auditory processing in human primary auditory cortex. J Neurosci 23:11516-11522. https://doi.org/10.1523/jneurosci.23-37-11516. 2003

Di S, Baumgartner C, Barth DS (1990) Laminar analysis of extracellular field potentials in rat vibrissa/barrel cortex. J Neurophysiol 63:832-840. https://doi.org/10.1152/jn.1990.63.4.832
Douglas RJ, Martin KAC (2004) Neuronal circuits of the neocortex. Annu Rev Neurosci 27:419-451. https://doi.org/10.1146/annurev. neuro.27.070203.144152

Eggermont JJ, Ponton CW (2002) The neurophysiology of auditory perception: from single units to evoked potentials. Audiol Neurootol 7:71-99. https://doi.org/10.1159/000057656

Elston GN, Benavides-Piccione R, DeFelipe J (2001) The pyramidal cell in cognition: a comparative study in human and monkey. $\mathrm{J}$ Neurosci 21:1-5. https://doi.org/10.1523/jneurosci.21-17-j0002. 2001

Fan CSD, Zhu X, Dosch HG et al (2017) Language related differences of the sustained response evoked by natural speech sounds. PLoS ONE 12:1-21. https://doi.org/10.1371/journal. pone. 0180441

Fischl B, Dale AM (2000) Measuring the thickness of the human cerebral cortex from magnetic resonance images. Proc Natl Acad Sci U S A 97:11050-11055. https://doi.org/10.1073/pnas.200033797

Friedman DP, Jones EG (1980) Focal projection of electrophysiologically defined groupings of thalamic cells on the monkey somatic sensory cortex. Brain Res 191:249-252. https://doi.org/10.1016/ 0006-8993(80)90328-5

Galaburda AM, LeMay M, Kemper TL, Geschwind N (1978) Right-left asymmetries in the brain. Science 199:852-856. https://doi.org/ 10.1126/science. 341314

Geyer S, Schleiches A, Zilles K (1997) The somatosensory cortex of human: cytoarchitecture and regional distributions of receptorbinding sites. Neuroimage 6:27-45. https://doi.org/10.1006/nimg. 1997.0271

Gilbert CD (1983) Microcircuitry of the visual cortex. Annu Rev Neurosci 6:217-247. https://doi.org/10.1146/annurev.ne.06.030183. 001245

Gilbert CD, Wiesel TN (1989) Columnar specificity of intrinsic horizontal and corticocortical connections in cat visual cortex. J Neurosci 9:2432-2422. https://doi.org/10.1523/jneurosci.09-0702432.1989

Goodin DS, Squires KC, Starr A (1978) Long latency event-related components of the auditory evoked potential in dementia. Brain 101:635-648. https://doi.org/10.1093/brain/101.4.635

Gross N, Small A, Thompson D (1967) Response to contralateral and ipsilateral auditory stimulation from the same cortical areas. Brain Res 158:951-958. https://doi.org/10.1126/science.158.3803.951

Hagen E, Næss S, Ness TV, Einevoll GT (2018) Multimodal modeling of neural network activity: computing LFP, ECoG, EEG, and MEG signals with LFPy 2.0. Front Neuroinform. https://doi. org/10.3389/fninf.2018.00092

Hämäläinen M, Hari R, Ilmoniemi RJ et al (1993) Magnetoencephalography theory, instrumentation, and applications to noninvasive studies of the working human brain. Rev Mod Phys 65:413-497. https://doi.org/10.1103/RevModPhys.65.413

Hine J, Debener S (2007) Late auditory evoked potentials asymmetry revisited. Clin Neurophysiol 118:1274-1285. https://doi.org/10. 1016/j.clinph.2007.03.012

Howard MF, Poeppel D (2009) Hemispheric asymmetry in mid and long latency neuromagnetic responses to single clicks. Hear Res 257:41-52. https://doi.org/10.1016/j.heares.2009.07.010

Huang CL, Winer JA (2000) Auditory thalamocortical projections in the cat: laminar and areal patterns of input. J Comp Neurol 427:302-331. https://doi.org/10.1002/1096-9861(20001113)427: $2<302::$ AID-CNE10>3.0.CO;2-J

Ikeda A, Shibasaki H (1992) Invasive recording of movement-related cortical potentials in humans. J Clin Neurophysiol 9:509-520

Jäncke L, Wüstenberg T, Schulze K, Heinze HJ (2002) Asymmetric hemodynamic responses of the human auditory cortex to monaural and binaural stimulation. Hear Res 170:166-178. https://doi. org/10.1016/S0378-5955(02)00488-4 
Ji XY, Zingg B, Mesik L et al (2016) Thalamocortical innervation pattern in mouse auditory and visual cortex: laminar and cell-type specificity. Cereb Cortex 26:2612-2625. https://doi.org/10.1093/ cercor/bhv099

Jones SR, Pritchett DL, Stufflebeam SM et al (2007) Neural correlates of tactile detection: a combined magnetoencephalography and biophysically based computational modeling study. J Neurosci 27:10751-10764. https://doi.org/10.1523/jneurosci.0482-07.2007

Jones SR, Pritchett DL, Sikora MA et al (2009) Quantitative analysis and biophysically realistic neural modeling of the MEG Mu rhythm: rhythmogenesis and modulation of sensory-evoked responses. J Neurophysiol 102:3554-3572. https://doi.org/10. 1152/jn.00535.2009

Kiebel SJ, Garrido MI, Moran R et al (2009) Dynamic causal modeling for EEG and MEG. Hum Brain Mapp 30:1866-1876. https://doi. org $/ 10.1002 / \mathrm{hbm} .20775$

Kimura D (1961) Some effects of temporal-lobe damage on auditory perception. Can J Psychol 15:156-165. https://doi.org/10.1037/ h0083218

Kimura D (2011) From ear to brain. Brain Cogn 76:214-217. https:// doi.org/10.1016/j.bandc.2010.11.009

Kulics AT, Cauller LJ (1986) Cerebral cortical somatosensory evoked responses, multiple unit activity and current source-densities: their interrelationships and significance to somatic sensation as revealed by stimulation of the awake monkey's hand. Exp Brain Res 62:46-60. https://doi.org/10.1007/BF00237402

Lee S, Jones SR (2013) Distinguishing mechanisms of gamma frequency oscillations in human current source signals using a computational model of a laminar neocortical network. Front Hum Neurosci 7:1-19. https://doi.org/10.3389/fnhum.2013.00869

Linden JF, Schreiner CE (2003) Columnar transformations in auditory cortex? A comparison to visual and somatosensory cortices. Cereb Cortex 13:83-89. https://doi.org/10.1093/cercor/13.1.83

Loveless N, Vasama JP, Mäkelä J, Hari R (1994) Human auditory cortical mechanisms of sound lateralisation: III. Monaural and binaural shift responses. Hear Res 81:91-99. https://doi.org/10. 1016/0378-5955(94)90156-2

Mäkelä JP, Hämäläinen M, Hari R, McEvoy L (1994) Whole-head mapping of middle-latency auditory evoked magnetic fields. Electroencephalogr Clin Neurophysiol Evoked Potentials 92:414-421. https://doi.org/10.1016/0168-5597(94)90018-3

Mitani A, Shimokouchi M (1985) Neuronal connections in the primary auditory cortex: an electrophysiological study in the cat. J Comp Neurol 235:417-429. https://doi.org/10.1002/cne.902350402

Mitani A, Shimokouchi M, Itoh K et al (1985) Morphology and laminar organization of electrophysiologically identified neurons in the primary auditory cortex in the cat. J Comp Neurol 235:430-447. https://doi.org/10.1002/cne.902350403

Mononen LJ, Seitz MR (1977) An AER analysis of contralateral advantage in transmission of auditory information. Neuropsychologia $15: 165-173$

Murakami S, Okada Y (2006) Contributions of principal neocortical neurons to magnetoencephalography and electroencephalography signals. J Physiol 575:925-936. https://doi.org/10.1113/jphysiol. 2006.105379

Murakami S, Hirose A, Okada YC (2003) Contribution of ionic currents to magnetoencephalography (MEG) and electroencephalography (EEG) signals generated by guinea-pig CA3 slices. J Physiol 553:975-985. https://doi.org/10.1113/jphysiol.2003.051144

Neymotin SA, Daniels DS, Caldwell B et al (2020) Human neocortical neurosolver $(\mathrm{HNN})$, a new software tool for interpreting the cellular and network origin of human MEG/EEG data. Elife 9:1-39. https://doi.org/10.7554/eLife.51214

Ojima H, Honda CN, Jones EG (1991) Patterns of axon collateralization of identified supragranular pyramidal neurons in the cat auditory cortex. Cereb Cortex 1:80-94. https://doi.org/10.1093/ cercor/1.1.80

Ojima H, Honda CN, Jones EG (1992) Characteristics of intracellularly injected infragranular pyramidal neurons in cat primary auditory cortex. Cereb Cortex 2:197-216. https://doi.org/10.1093/cercor/ 2.3.197

Okada YC, Wu J, Kyuhou S (1997) Genesis of MEG signals in a mammalian CNS structure. Electroencephalogr Clin Neurophysiol 103:474-485. https://doi.org/10.1016/S0013-4694(97)00043-6

Oviedo HV (2017) Connectivity motifs of inhibitory neurons in the mouse auditory cortex. Sci Rep 7:1-9. https://doi.org/10.1038/ s41598-017-16904-2

Pandya DN, Rosene DL (1993) Laminar termination patterns of thalamic, callosal, and association afferents in primary auditory area of the rhesus monkey. Exp Neurol 119:220-234

Pantev C, Lütkenhöner B, Hoke M, Lehnertz K (1986) Comparison between simultaneously recorded auditory-evoked magnetic fields and potentials elicited by ipsilateral, contralateral and binaural tone burst stimulation. Int J Audiol 25:54-61. https://doi.org/10. 3109/00206098609078369

Pantev C, Ross B, Berg P et al (1998) Study of the human auditory cortices using a whole-head magnetometer: left vs. right hemisphere and ipsilateral vs. contralateral stimulation. Audiol Neurotol 3:183-190. https://doi.org/10.1159/000013789

Parviainen T, Helenius P, Salmelin R (2005) Cortical differentiation of speech and nonspeech sounds at $100 \mathrm{~ms}$ : implications for dyslexia. Cereb Cortex 15:1054-1063. https://doi.org/10.1093/cercor/ bhh206

Parviainen T, Helenius P, Salmelin R (2019) Children show hemispheric differences in the basic auditory response properties. Hum Brain Mapp 40:2699-2710. https://doi.org/10.1002/hbm.24553

Paulraj MP, Subramaniam K, Yaccob S, Bin et al (2015) Auditory evoked potential response and hearing loss: a review. Open Biomed Eng J 9:17-24. https://doi.org/10.2174/1874120701 509010017

Penhune VB, Zatorre RJ, MacDonald JD, Evans AC (1996) Interhemispheric anatomical differences in human primary auditory cortex: probabilistic mapping and volume measurement from magnetic resonance scans. Cereb Cortex 6:661-672. https://doi.org/10. 1093/cercor/6.5.661

Peronnet F, Michel F, Echallier JF, Girod J (1974) Coronal topography of human auditory evoked responses. Electroencephalogr Clin Neurophysiol 37:225-230. https://doi.org/10.1016/0013-4694(74) 90025-X

Petkov CI, Kang X, Alho K et al (2004) Attentional modulation of human auditory cortex. Nat Neurosci 7:658-663. https://doi.org/ $10.1038 / \mathrm{nn} 1256$

Picton TW, Hillyard SA, Krausz HI, Galambos R (1974) Human auditory evoked potentials. I: evaluation of components. Electroencephalogr Clin Neurophysiol 36:179-190. https://doi.org/10.1016/ 0013-4694(74)90155-2

Picton TW (2011) Human auditory evoked potentials. Plural Publishing, Abingdon

Rademacher J, Rademacher J, Caviness VS et al (1993) Topographical variation of the human primary cortices: implications for neuroimaging, brain mapping, and neurobiology. Cereb Cortex 3:313-329. https://doi.org/10.1093/cercor/3.4.313

Reite M, Zimmerman JT, Zimmerman JE (1981) Magnetic auditory evoked fields: interhemispheric asymmetry. Electroencephalogr Clin Neurophysiol 51:388-392. https://doi.org/10.1016/00134694(81)90102-4

Rockland KS, Pandya DN (1979) Laminar origins and terminations of cortical connections of the occipital lobe in the rhesus monkey. Brain Res 179:3-20. https://doi.org/10.1016/0006-8993(79) 90485-2 
Rosenzweig R (1951) Representations of the two ears at the auditory cortex. Am J Physiol 167:147-158

Sacchet MD, LaPlante RA, Wan Q et al (2015) Attention drives synchronization of alpha and beta rhythms between right inferior frontal and primary sensory neocortex. J Neurosci 35:2074-2082. https://doi.org/10.1523/JNEUROSCI.1292-14.2015

Sakata S, Harris KD (2009) Laminar structure of spontaneous and sensory-evoked population activity in auditory cortex. Neuron 64:404-418. https://doi.org/10.1016/j.neuron.2009.09.020

Salmelin R, Schnitzler A, Parkkonen L et al (1999) Native language, gender, and functional organization of the auditory cortex. Proc Natl Acad Sci U S A 96:10460-10465. https://doi.org/10.1073/ pnas.96.18.10460

Samatra DPGP, Meliana G, Purna Putra IGNP, Widyadharma IPE (2020) The difference of brainstem auditory evoked potential latency idiabetic patient with good and poor glycemic control. Open Access Maced J Med Sci 8:457-462. https://doi.org/10. 3889/oamjms.2020.3403

Sanz Leon P, Knock SA, Woodman MM et al (2013) The virtual brain: a simulator of primate brain network dynamics. Front Neuroinform. https://doi.org/10.3389/fninf.2013.00010

Schönwiesner M, Krumbholz K, Rübsamen R et al (2007) Hemispheric asymmetry for auditory processing in the human auditory brain stem, thalamus, and cortex. Cereb Cortex 17:492-499. https://doi. org/10.1093/cercor/bhj165

Schroeder CE, Seto S, Arezzo JC, Garraghty PE (1995) Electrophysiological evidence for overlapping dominant and latent inputs to somatosensory cortex in squirrel monkeys. J Neurophysiol 74:722-731. https://doi.org/10.1152/jn.1995.74.2.722

Shahin A, Bosnyak DJ, Trainor LJ, Roberts LE (2003) Enhancement of neuroplastic $\mathrm{P} 2$ and $\mathrm{N} 1 \mathrm{c}$ auditory evoked potentials in musicians. J Neurosci 23:5545-5552. https://doi.org/10.1523/jneurosci.2313-05545.2003

Shaw ME, Hämäläinen MS, Gutschalk A (2013) How anatomical asymmetry of human auditory cortex can lead to a rightward bias in auditory evoked fields. Neuroimage 74:22-29. https://doi.org/ 10.1016/j.neuroimage.2013.02.002

Sherman MA, Lee S, Law R et al (2016) Neural mechanisms of transient neocortical beta rhythms: converging evidence from humans, computational modeling, monkeys, and mice. Proc Natl Acad Sci 113:E4885-E4894. https://doi.org/10.1073/pnas.1604135113

Shin H, Law R, Tsutsui S et al (2017) The rate of transient beta frequency events predicts behavior across tasks and species. Elife 6:1-31. https://doi.org/10.7554/eLife.29086
Sliva DD, Black CJ, Bowary P et al (2018) A Prospective Study of the Impact of Transcranial Alternating Current Stimulation on EEG Correlates of Somatosensory Perception. Front Psychol 9:2117. https://doi.org/10.3389/fpsyg.2018.02117

Stephen JM, Hill DE, Peters A et al (2017) Development of auditory evoked responses in normally developing preschool children and children with autism spectrum disorder. Dev Neurosci 39:430 441. https://doi.org/10.1159/000477614

Taub JM, Tanguay PE, Doubleday CN et al (1976) Hemisphere and ear asymmetry in the auditory evoked response to musical chord stimuli. Physiol Psychol 4:11-17. https://doi.org/10.3758/BF033 26537

Tunturi AR (1946) A study on the pathway from the medial geniculate body to the acoustic cortex in the dog. Am J Physiol 147:311-319

van Bijnen S, Kärkkäinen S, Helenius P, Parviainen T (2019) Left hemisphere enhancement of auditory activation in language impaired children. Sci Rep 9:1-11. https://doi.org/10.1038/ s41598-019-45597-y

Wagner M, Lee J, Mingino F et al (2017) Language experience with a native-language phoneme sequence modulates the effects of attention on cortical sensory processing. Front Neurosci. https://doi. org/10.3389/fnins.2017.00569

Wallace MN, Palmer AR (2008) Laminar differences in the response properties of cells in the primary auditory cortex. Exp Brain Res 184:179-191. https://doi.org/10.1007/s00221-007-1092-z

Wolpaw JR, Penry JK (1977) Hemispheric differences in the auditory evoked response. Electroencephalogr Clin Neurophysiol 43:99102. https://doi.org/10.1016/0013-4694(77)90200-0

Yoshiura T, Ueno S, Iramina K, Masuda K (1994) Effects of stimulation side on human middle latency auditory evoked magnetic fields. Neurosci Lett 172:159-162

Ziegler DA, Pritchett DL, Hosseini-Varnamkhasti P et al (2010) Transformations in oscillatory activity and evoked responses in primary somatosensory cortex in middle age: a combined computational neural modeling and MEG study. Neuroimage 52:897-912. https://doi.org/10.1016/j.neuroimage.2010.02.004

Publisher's note Springer Nature remains neutral with regard to jurisdictional claims in published maps and institutional affiliations. 